\title{
Tool-Wear Monitoring Based on Continuous Hidden Markov Models
}

\author{
Antonio G. Vallejo Jr. ${ }^{1}$, Juan A. Nolazco-Flores ${ }^{2}$, Rubén Morales-Menéndez ${ }^{2}$, \\ L. Enrique Sucar ${ }^{3}$, and Ciro A. Rodríguez ${ }^{2}$ \\ ${ }^{1}$ ITESM Laguna Campus, Mechatronic Dept., Torreón, Coah., México \\ 2 ITESM Monterrey Campus, Monterrey NL, México \\ 3 ITESM Morelos Campus, Cuernavaca Mor, México \\ \{avallejo, jnolazco, rmm, esucar, ciro.rodriguez\}@itesm.mx
}

\begin{abstract}
In this work we propose to monitor the cutting tool-wear condition in a CNC-machining center by using continuous Hidden Markov Models (HMM). A database was built with the vibration signals obtained during the machining process. The workpiece used in the milling process was aluminum 6061. Cutting tests were performed on a Huron milling machine equipped with a Sinumerik 840D open CNC. We trained/tested the HMM under 18 different operating conditions. We identified three key transitions in the signals. First, the cutting tool touches the workpiece. Second, a stable waveform is observed when the tool is in contact with the workpiece. Third, the tool finishes the milling process. Considering these transitions, we use a five-state HMM for modeling the process. The HMMs are created by preprocessing the waveforms, followed by training step using Baum-Welch algorithm. In the recognition process, the signal waveform is also preprocessed, then the trained HMM are used for decoding. Early experimental results validate our proposal in exploiting speech recognition frameworks in monitoring machining centers. The classifier was capable of detecting the cutting tool condition within large variations of spindle speed and feed rate, and accuracy of $84.19 \%$.
\end{abstract}

Keywords: Signal Processing and Analysis, Remote Sensing Applications of Pattern Recognition, Hidden Markov Models, Tool-wear monitoring.

\section{Introduction}

Manufacturing processes are typically complex. High Speed Machining (HSM) systems demand precise and complex operations; operators have to implement complicated operations in these systems too. Computerized numerical controls (CNC) systems demand supervisor and protection functions such as monitoring, and supervising 5. Also, special software for supporting operators is required [7.

In any typical metal-cutting process, key factors that define the product quality are dimensional accuracy and surface finish. One important part in the 
CNC machines is the cutting tool condition, and it is important to constraint the following aspects: progressive tool wear, deflection of cutting tool and the variation of process conditions. We need a cutting tool condition monitoring system in order to reduce operating cost with the same quality, [13.

Tool wear is caused by a combination of various phenomena. Contact with the chip produces a crater in the face of the tool. Flank wear, on the other hand, is commonly due to friction between the tool and the work-piece material. Once the protective coating is removed, sudden chipping of the cutting edges may occur, leading to catastrophic failure of the tool. Recent studies conclude that rake-face wear, flank wear, chipping and breakage are the main modes of tool wear in HSM. One of the main goals in HSM is to find an appropriate trade-off among tool wear, surface quality and productivity, considering the cost of the tool, its replacement cost, the cost of maintain the machine in idle time, and so forth.

Safety is fundamental in tool condition monitoring systems; also, accurate data acquisition from sensors are mandatory. Sensors should meet certain requirements ensuring robustness, reliability and non-intrusive behavior under normal working conditions. Almost all sensors present restrictions in the manufacturing industry because the harsh environment. The development of new sensors or technologies for monitoring tool wear are critical in machining business.

In this work, we propose a new recognition approach for tool-wear monitoring using continuous Hidden Markov Models (HMM). The vibration signals between the tool and the workpiece will provide the database. In section 2, we describe the state of the art. In section 3 we present our proposal to solve the problem. In section 4, the experimental set up is described. In section 5, the experimental results are shown. Finally, section $[$ concludes the paper.

\section{State of the Art}

Tool failure represents about $20 \%$ of machine tool down-time, and tool wear negatively impacts the work quality in the context of dimensions, finish, and surface integrity 9]. Using fuzzy logic, artificial neural networks, and linear regression, important contributions for tool-wear monitoring had been proposed, with different sensors (acoustic, electrical, magnetic, accelerometer, etc.) installed in strategic points of the CNC machine.

In [5], Haber and Alique developed an intelligent supervisory system for tool wear prediction using a model-based approach. In order to deal with nonlinear process characteristics, they used an Artificial Neural Network (ANN) output error model to predict online the resultant cutting force under different cutting conditions. First, an ANN model is created considering the cutting force, the feed rate, and the radial depth of the cut. The residual error obtained of the two forces was compared with an adaptive threshold to estimate the tool wear. This method evaluated the behavior of the tool in three states; new tool, half-worn tool, and worn tool.

In [6], Haber et al. presented an investigation of tool wear monitoring in a high speed machining process on the basis of the analysis of different sig- 
nals signatures in the time and frequency domains. They used sensorial information from relevant sensors (e.g., dynamometer, accelerometer, and acoustic emission sensor) to obtain the deviation of representative variables. During the tests measurements at different cutting speeds and feed rates were carried out to determine the effects of a new and worn tool in high speed roughing. Data were transformed from time domain to frequency domain through a Fast Fourier Transformer (FFT) algorithm in order to analyze frequency components. They conducted second harmonic of the tooth path excitation frequency in the vibration signal is the best index for tool wear monitoring. Additionally, the spectrum analysis of acoustic emission (AE) signals corroborates that AE sensors are very sensitive to changes in tool condition. Also, [13] worked with multilayered neural networks for tool condition monitoring in the milling process.

In [10, Owsley et al. presented an approach for feature extraction from vibrations during the drilling. Self-organizing feature maps (SOFM's) extract the features. They modified the SOFM algorithm in order to improve its generalization abilities and to allow it to server as a preprocessor for a HMM classifier. The authors used a discrete hidden Markov model. Similar proposals for tool-wear monitoring can be found in [215/1814].

\section{Tool Wear Monitoring System}

Figure 1 shows a flow diagram of the system for monitoring tool-wear using continuous HMM.

The vibration signal in the machining process is considered the input signal. As we can see in Figure 1, the input signal is preprocessed and then it is separated into two branches. The training data branch leads to a HMM model. Given the model and the parameterized signal a decoder produce a transcript of a specific pattern as a result. In this training phase the system learns the patterns that

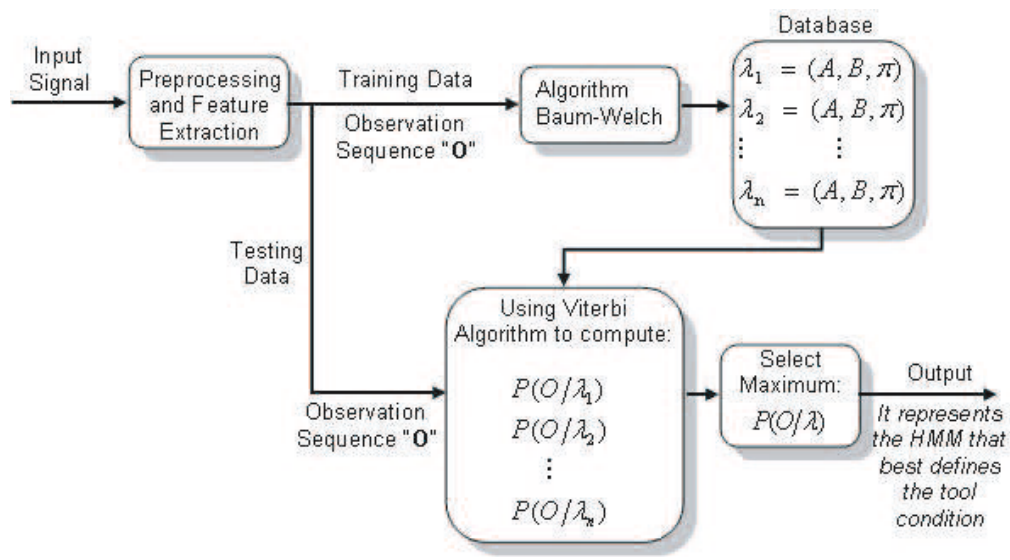

Fig. 1. Flow diagram for monitoring the tool-wear with continuous HMM 
represent the vibration signals. The testing branch uses the preprocessed input signal and the HMM model to compute $P(O \mid \lambda)$ using the Viterbi algorithm for each model. The model with higher probability is selected as result. Next, we review the steps and basic concepts of the proposed algorithm.

\subsection{Hidden Markov Models}

Real world processes produce observable outputs which can be characterized as signals (discrete/continuous, pure/corrupted, etc.). A problem of fundamental interest is characterizing such signals in terms of models (deterministic/statistical). Statistical models use the statistical properties of the signal, such as Hidden Markov Models, 113.

Definitions. For completeness we will review some basic definitions. A HMM, as depicted in Figure 2, is characterized by the following:

- $N$, number of states in the model. We denote the states as $S=S_{1}, \cdots, S_{N}$, and the state at time $t$ as $q_{t}$.

- $M$, number of distinct observation symbols per state. We denote the individual symbols as $V=v_{1}, \cdots, v_{M}$.

- The state transition probability distribution $A=P\left[q_{t}=S_{j} \mid q_{t-1}=S_{i}\right], 1 \leq i j \leq N$

- The observation symbol probability distribution in state $j$, $B=P\left[v_{k} \mid q_{t}=S_{j}\right], 1 \leq j \leq N, 1 \leq k \leq M$

- The initial state distribution $\pi=P\left[q_{1}=S_{i}\right], 1 \leq i \leq N$

Given appropriate values of $N, M, A, B$, and $\pi$, the HMM can be used as a generator to give an observation sequence $O=O_{1}, \cdots, O_{T}$. Then, a complete specification of an HMM requires specification of two model parameters $(N$,

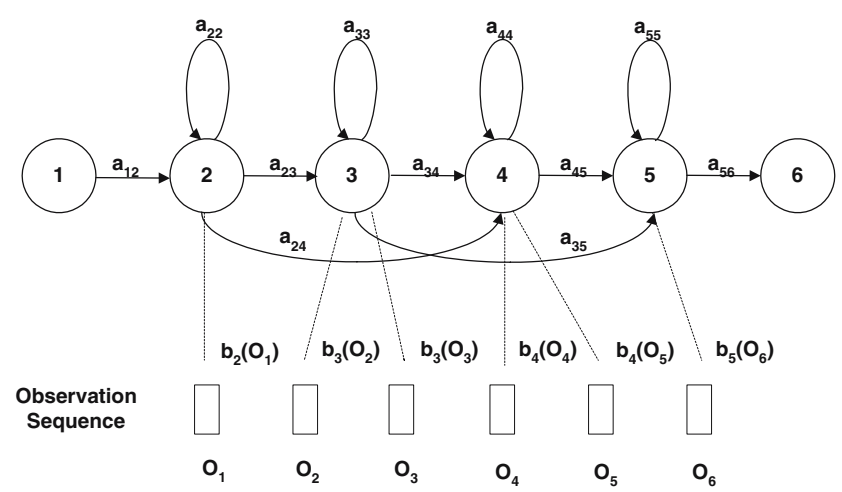

Fig. 2. Elements of a HMM: left-right model, 6 states, and observations per state 
and $M)$, specification of observation symbols, and the specification of the three probability measures $\lambda=(A, B, \pi)$. The parameters $N, M$ and $\lambda$ are learned from data. Given this model and the observation we can compute $P(O \mid \lambda)$.

\subsection{Baum-Welch Algorithm}

The well-known Baum-Welch algorithm [1] is used to compute the model parameters (means, variance, and transitions) given the training data. It is an iterative process for parameter estimation based on a training data set for a given model $\lambda$. The goal is to obtain a new model $\bar{\lambda}$ where the function

$$
Q(\lambda, \bar{\lambda})=\sum_{Q} \frac{P(O, Q \mid \lambda)}{P(O \mid \lambda)} \log [P(O, Q \mid \bar{\lambda})]
$$

is maximized. For this algorithm it is need to define a forward and a backward probability as

$$
\alpha_{t}(i)=P\left(O_{1}^{t}, q_{t}=i \mid \lambda\right), \quad \beta_{t}(i)=P\left(O_{t+1}^{T} \mid q_{t}=i, \lambda\right)
$$

Based on this two functions, the probability for changing from state $j$ to state $k$ at time $t$ can be defined as

$$
\xi_{t}(j, k)=\frac{\sum_{i} \alpha_{i-1}(i) a_{i j} c_{j k} b_{j k}\left(o_{t}\right) \beta_{t}(j)}{\sum_{i=1}^{N} \alpha_{T}(i)}
$$

where $b_{j}(o)$ is a continuous output probability density function (pdf) for state $j$ and can be described as a weighted mixture of Gaussian functions, as follow

$$
b_{j}(o)=\sum_{k=1}^{M} c_{j k} N\left(o, \mu_{j k}, U_{j k}\right)=\sum_{k=1}^{M} c_{j k} b_{j k}\left(o, \mu_{j k}, U_{j k}\right)
$$

where $c_{j k}$ is the weight of the gaussian $k$ and $N\left(o, \mu_{j k}, U_{j k}\right)$ is a single gaussian of mean value $\mu_{j k}$ and a covariance matrix $U_{j k}$. Therefore, the model can be described in terms of $\mu_{j k}, U_{j k}$ and $c_{j k}$, and the new set of parameters for model $\bar{\lambda}$ are recalculated using Baum-Welch as follow

$$
\begin{gathered}
\bar{\mu}_{j k}=\frac{\sum_{t=1}^{T} \xi(j, k) o_{t}}{\sum_{t=1}^{T} \xi_{t}(t, k)} \\
\bar{U}_{j k}=\frac{\sum_{t=1}^{T} \xi_{t}(j, k)\left(o_{t}-\bar{\mu}_{j k}\right)\left(o_{t}-\bar{\mu}_{j k}\right)^{t}}{\sum_{t=1}^{T} \xi_{t}(j, k)} \\
\bar{c}_{j k}=\frac{\sum_{t=1}^{T} \xi_{t}(j, k)}{\sum_{t=1}^{T} \sum_{k} \xi_{t}(j, k)}
\end{gathered}
$$

Now, the term $b_{j k}$ can be written as

$$
b_{j k}\left(o_{t}, \mu_{j k}, \sigma_{j k}\right)=\frac{1}{\prod_{i=1}^{d} \sqrt{2 \pi \sigma_{j k i}}} e^{-\frac{1}{2} \sum_{i=1}^{d}\left(\frac{o_{t i}-\mu_{j k i}}{\sigma_{j k i}}\right)^{2}}
$$




\subsection{Viterbi Algorithm}

The Viterbi algorithm 3 is used to find the single best state sequence, $Q=$ $q_{1}, \cdots, q_{T}$, for the given observation sequence $O=O_{1}, \cdots, O_{T}$, we need to define the quantity

$$
P(O \mid \lambda)=\max _{q_{1}, \cdots, q_{t-1}} P\left[q_{1}, \cdots, q_{t}=i, O_{1}, \cdots, O_{t} \mid \lambda\right]
$$

\subsection{Feature Extraction}

The vibration signals are pre-processed calculating their Mel Frequency Cesptral Coefficient (MFCC) representation [12. This common transformation has shown to be more robust and reliable than other techniques. The process to calculate the MFCC is shown in Figure 3 .

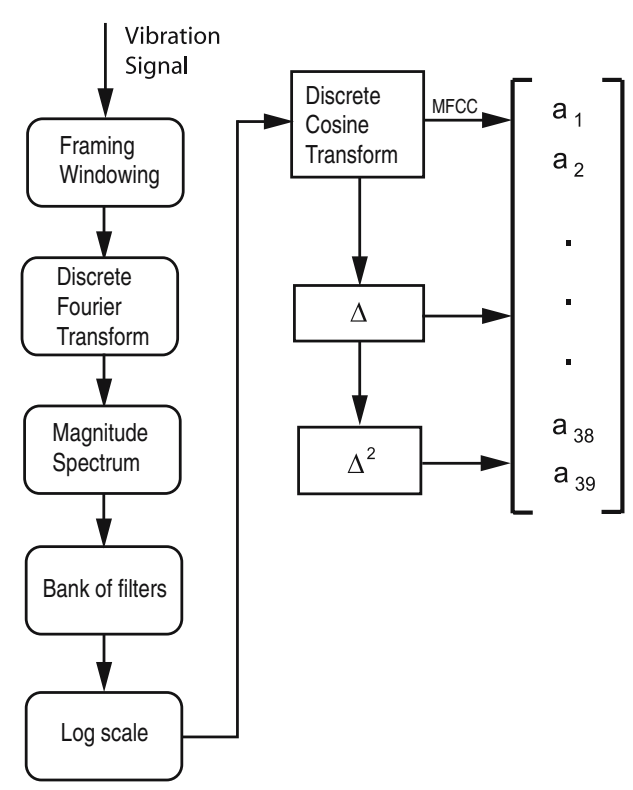

Fig. 3. Feature extraction process

Each signal is divided into short frames. For each frame the amplitude spectrum is obtained using the Discrete Fourier Transform (DFT). Afterwards, the spectrum is converted to a logarithm scale. To smooth the scaled spectrum, bandpass filter banks are used. Finally, the discrete cosine transform is applied to eliminate the correlation between components. The result is a 13-dimension vector, each dimension corresponding to one parameter. We applied similar considerations as in speech recognition [4, where it is common to estimate the timederivative $(\Delta)$ and the time-acceleration $\left(\Delta^{2}\right)$ of each parameter. Then, the final 
representation is a 39 dimension vector formed by 12-dimension MFCC, followed by 1 energy coefficient, $13 \Delta$ and $13 \Delta^{2}$ coefficients.

\section{Experimental Set Up}

\subsection{CNC Machine}

The experimental tests were conducted in a KX10 HURON machining center, with a capacity of $20 \mathrm{KW}$, three axis, and equipped with a SIEMENS openSinumerik 840D controller, left image in Figure 4. This machining center possesses high precision sideways that allow all three axis to reach a speed of up to $30 \mathrm{~m} / \mathrm{min}$. The machine has high-rigidity, high-precision features and there is not interference between the workpiece and the moving parts.
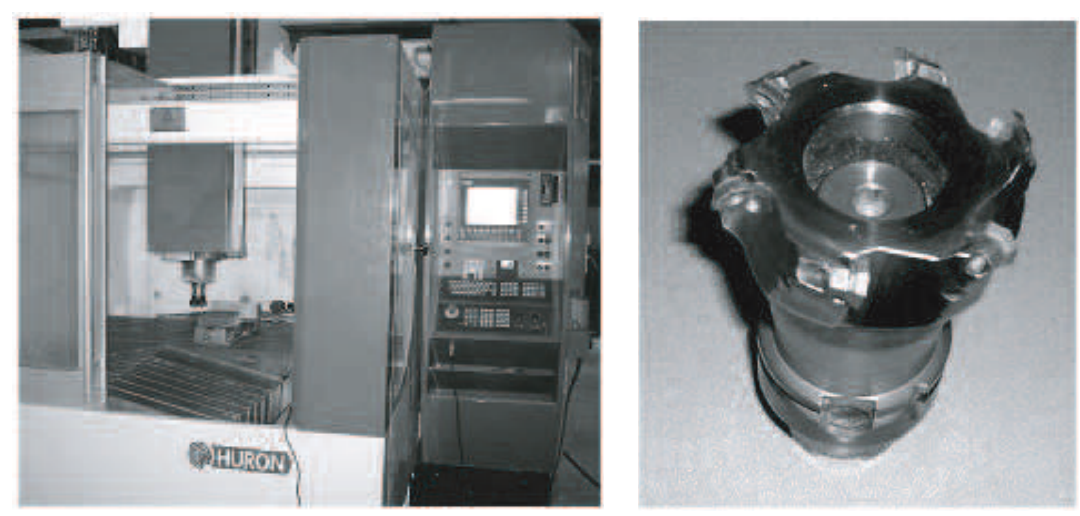

Fig. 4. KX10 Huron CNC-milling center (left), and cutting tool (right)

The cutting tool is an Octomill R220.43-03.00-05 face mill of SECO Carboloy, with a diameter of $80 \mathrm{~mm}$, depth of cut $3.5 \mathrm{~mm}$, and six inserts of the SECO Carboloy OFEX-05T305TN-ME07 T250M type, right image in Figure 4.

\subsection{Data Acquisition System}

Figure 5 shows a diagram of the experimental set-up. The vibration signal is recorded by using an accelerometer installed on the flat metal support. The vibration signals during the machining process was acquired using a 8 bits analogdigital converter (ADC) sampling at $50 \mathrm{KHz}$.

The accelerometer has as sensing element a ceramic/shear with $( \pm 20 \%) 10.2$ $\mathrm{mV} /\left(\mathrm{m} / \mathrm{s}^{2}\right)$ sensitivity and a frequency range of $1.2 \mathrm{~Hz}-10 \mathrm{KHz}$. The range of measurement is $\pm 490 \mathrm{~m} / \mathrm{s}^{2}$. We recorded the vibration signals for several machining conditions. Spindle speed : 2,000, 1,500, and 1,000 rev/min. Feed 


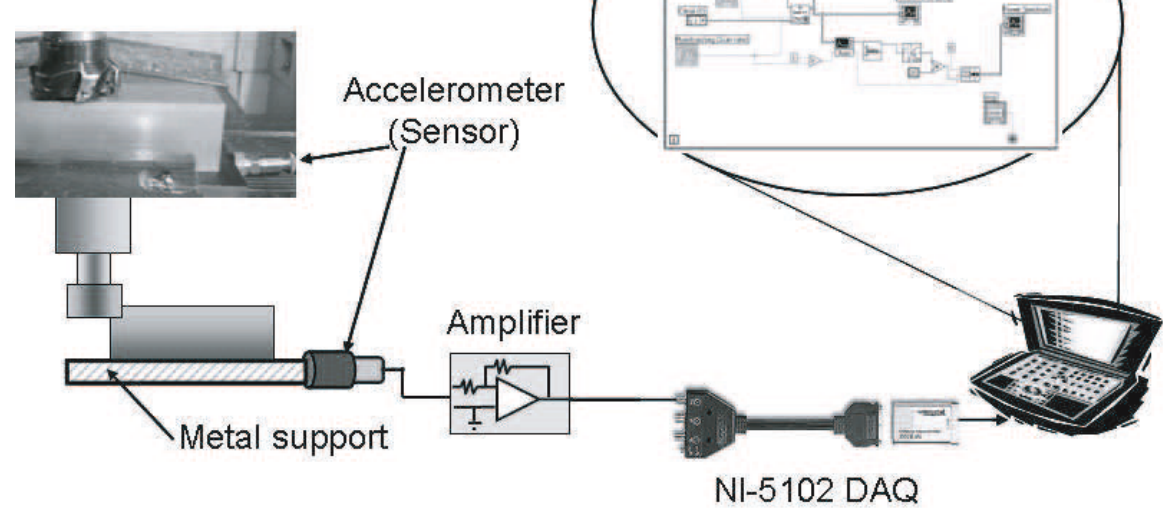

Fig. 5. Experimental Set-up: Acquisition System to record the vibration signals

rate of the tool : 600,800 , and $1,000 \mathrm{~mm} / \mathrm{min}$. Depth of the tool: $1 \mathrm{~mm}$. All the experiments were made considering two conditions for the tool: good and worn inserts. We applied a full factorial design to consider all the defined levels for each machining condition. Then, we required 18 different operating conditions, and we reproduced the experiments 9 (tool with good inserts) and 8 (tool with worn inserts) times. We obtained 153 experiments. Figure 6] shows some examples of the vibration signals. The vibration signals on left of the figure represent normal conditions of the tool (inserts in good conditions) at different operating conditions. The vibration signals on the right of the figure were recorded with worn inserts.

\section{Results}

Our database was built with the vibration signals obtained during the machining process. This database contains 153 experiments under 18 different operating conditions. The first 5 experiments $\left(T_{r}\right)$ were used for training, and last 4 experiments were used for testing $\left(T_{s}\right)$. The data streams are processed using the Sphinx HMM Toolkit developed at Carnegie Mellon University. The toolkit was configured to use several Gaussian, left-right, five states, HMMs. Table 1 presents the accuracy when a signal is processed for the classifier.

We evaluate the performance of the classifier considering the following conditions: 

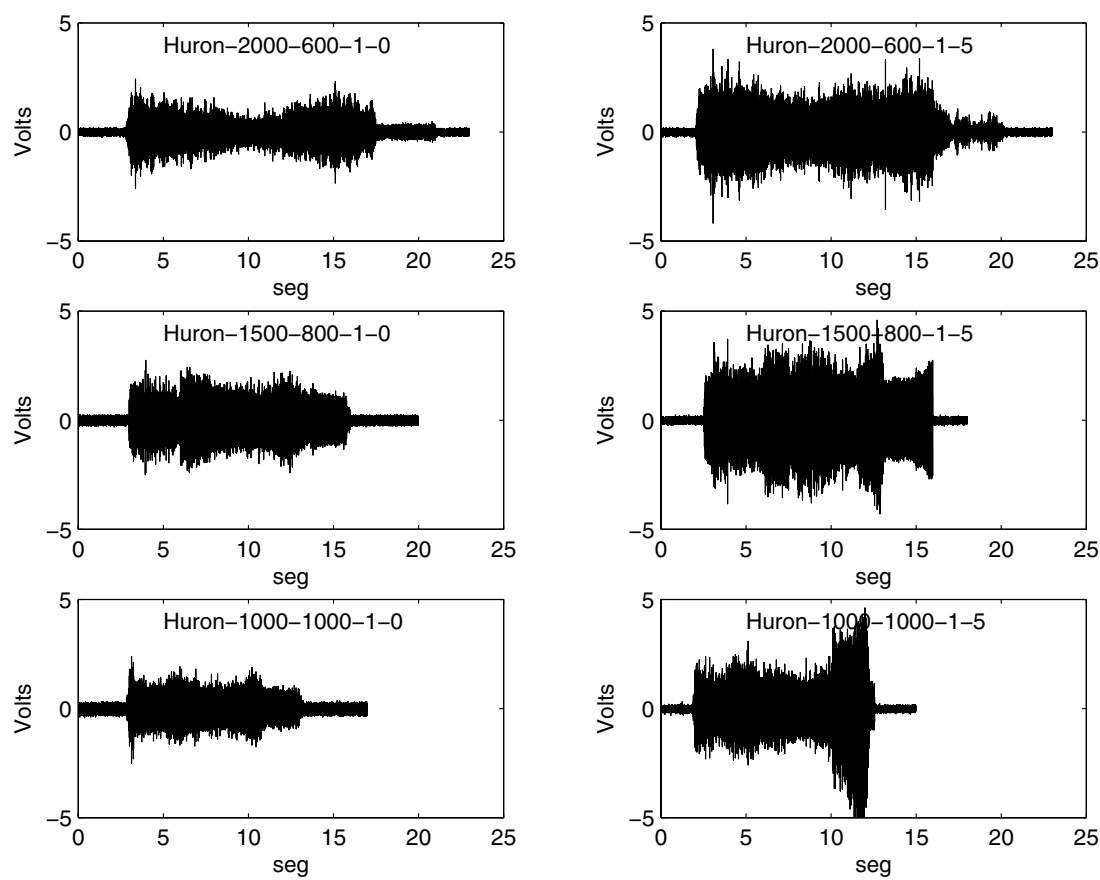

Fig. 6. Vibration Signals. Left signals represent good cutting tool conditions. Right signals were obtained with worn inserts. Each condition is defined by the spindle speed $(2000,1500,1000 \mathrm{rpm})$, feed rate $(600,800,1000 \mathrm{~mm} / \mathrm{min})$, depth of the cut $(1 \mathrm{~mm})$ and number of damage inserts $(0,5)$.

- First, we train and test the algorithm with the same database, $T_{r}=T_{s}$, and we obtain $95 \%$ of success, almost all conditions were identified. Note that we have very few data for both training and testing steps.

- Second, we test the algorithm with different database, $T_{r} \neq T_{s}$. We obtain $66.70 \%$ of success to recognize the pattern. In this case, the parameters of the HMM were obtained using only one Gaussian.

- Third, we compute the parameters of the HMM with different Gaussian. We obtained an $84.10 \%$ success with 16 Gaussian. We train and test the algorithm with different database.

We also configured the HMM toolkit for recognition of two states: good and faulty(worn inserts) condition. Table 2 presents the results for each condition using the HMMs with 16 Gaussian. This table also shows the False Alarm Rate (FAR) and the False Fault Rate (FFR) and Expected number of workpieces machining when the fault condition is detected. The FAR is the rate when decoder detects the tool is in fault condition (worn inserts), but the tool is in good condition. The FFR is the rate when decoder detects the tool is in good condition and it is not. The FAR condition is not a problem for the machining process. 
Table 1. Accuracy of the model

\begin{tabular}{|c|c|}
\hline Experiments for testing & Accuracy \\
\hline$T r=T s$ & $95 \%$ \\
\hline$T r \neq T s$ & $66.70 \%$ with 1 Gaussian \\
\hline$T r \neq T s$ & $68.30 \%$ with 2 Gaussian \\
\hline$T r \neq T s$ & $79.40 \%$ with 8 Gaussian \\
\hline$T r \neq T s$ & $84.10 \%$ with 16 Gaussian \\
\hline
\end{tabular}

However, the FFR condition could be a huge problem when it presents a higher value, because the poor quality of the product and the tool can be broken before being detected.

Given the FFR we can easily obtain the probability to detect the fault condition as follow:

$$
E(k)=\sum_{k=1}^{\infty} P(k)
$$

From this equation, we can also obtain the expected number of pieces processed before the fault condition is detected, as shown:

$$
E[k]=\frac{1}{1-P_{b, g}}
$$

This value is important because it establishes the number of pieces before the fault condition is detected. This number must be small to reduce the number of pieces with a poor quality surface, and to reduce the possibilities that the tool could be broken.

Table 2. Probabilities of the HMMs with the 16 gaussian

\begin{tabular}{|c|c|c|}
\hline Condition & Probability & Description \\
\hline$P_{g, g}, P_{b, b}$ & 0.841 & Success probability \\
\hline$P_{g, b}$ & 0.016 & False alarm rate $E(k)=1.016$ \\
\hline$P_{b, g}$ & 0.143 & False fault rate $E(k)=1.167$ \\
\hline
\end{tabular}

\section{Conclusions and Future Work}

In this paper we have proposed an algorithm to monitor the cutting tool-wear condition in a CNC-machining center by using continuous Hidden Markov Models. The speech recognition framework was exploited in this domain with successful results and great potential. A database was built with the vibration signals of different conditions during the machining process of an Aluminium 6061 workpiece. We trained/tested the HMM for each operating conditions, and the results were satisfactory given the limited number of experiments. This is a first stage in 
the development of an intelligent system to monitor, supervise, and control the machining process for a CNC-machining center. We are working in the process to acquire more vibration signals with other sensors installed in different points of the machine. We will use these additional signals to train and test new continuous HMMs and evaluate the accuracy of the classifier with the new conditions.

\section{References}

1. O B Abouelatta and J Madl. Surface roughness prediction based on cutting parameters and tool vibrations in turning operations. Materials Processing Technology, (118):269-277, 2001.

2. L Atlas, M Ostendorf, and G D Bernard. Hidden markov models for monitoring machining tool-wear. IEEE, pages 3887-3890, 2000.

3. Jeff Bilmes. What hmms can do. Technical report.

4. Steven B. Davis and Paul Mermelstein. Comparison of parametric representations for monosyllabic word recognition in continuously spoken sentences. IEEE Transactions on Acoustic, Speech, and Signal Processing, 4(28):357-366, 1980.

5. R E Haber and A Alique. Intelligent process supervision for predicting tool wear in machining processes. Mechatronics, (13):825-849, 2003.

6. R E Haber, J E Jiménez, C R Peres, and J R Alique. An investigation of toolwear monitoring in a high-spped machining process. Sensors and Actuators A, (116):539-545, 2004.

7. Y Koren, U Heisel, F Jovane, T Moriwaki, G Pritschow, G Ulsoy, and H Van Brussel. Reconfigurable manufacturing systems. Annals of the CIRP, 48(2):527540, 1999.

8. K Y Lee, M C Kang, Y H Jeong, D W Lee, and J S Kim. Simulation of surface roughness and profile in high-speed and milling. Materials Processing Technology, (113):410-415, 2001.

9. S Y Liang, R L Hecker, and R G Landers. Machining process monitoring and control: The state-of-the-art. ASME International Mechanical Engenieering Congress and Exposition, pages 1-12, 2002.

10. L M D Owsley, L E Atlas, and G D Bernard. Self-organizing feature maps and hidden markov models for machine-tool monitoring. IEEE Transactions on Signal Processing, 45(11):2787-2798, 1997.

11. L R Rabiner. A tutorial on hidden markov models and selected applications in speech recognition. Proceedings of the IEEE, 77(2):257-286, 1989.

12. L R Rabiner and B H Juang. Fundamentals of speech recognition. Prentice-Hall, New-Jersey, 1993.

13. H Saglam and A Unuvar. Tool condition monitoring in milling based on cutting forces by a neural network. International Journal of Production Research, 41(7):1519-1532, 2003.

14. Y H Tsai, J C Chen, and S J Lou. An in-process surface recognition system based on neural networks in end milling cutting operations. Machine Tools and Manufacture, (39):583-605, 1999.

15. G M Zhang and C Lin. A hidden markov model approach to the study of random tool motion during machining. Technical report. 